\title{
DNA Double Strand Breaks Occur Independent of AID in Hypermutating Ig Genes
}

\author{
LINDA BROSS and HEINZ JACOBS*
}

\author{
Department Immunology, University of Maastricht, Research Institute Growth and Development, Universiteits Singel 50, 6200 MD Maastricht, \\ The Netherlands
}

\begin{abstract}
Somatic hypermutation (SHM) and class switch recombination (CSR) take place in B cells of the germinal center (GC) and are associated with DNA double-strand breaks (DNA-DSBs). Transcription favors the generation of DNA-DSBs in the V-regions and switch regions of Ig genes. Both SHM and CSR are controlled by the Activation Induced Cytidine Deaminase (AID), an enzyme exclusively expressed in B cells of the GC. Because AID is capable of deaminating deoxy-cytidine (dC) to deoxy-uracil (dU), it might directly induce nicks (single strand DNA breaks) and also DNA-DSBs via a U-DNA glycosylase mediated base excision repair pathway ('DNA-substrate model'). Alternatively, AID could function like its closest homologue Apobec-1 as a catalytic subunit of a RNA editing holoenzyme ('RNA-substrate model'). To determine whether AID lies upstream or downstream of the DNA lesions found in hypermutating Ig genes, we have analysed the V $\lambda$ locus of AID proficient and AID deficient GC B cells for the presence of DNA-DSBs. Although rearranged V $\lambda$ genes are preferred targets of SHM we find that AID-proficient and -deficient V $\lambda 1 / 2$-expressing GC B cells display a similar frequency, distribution and sequence preference of DNA-DSBs in rearranged and germline $\mathrm{V} \lambda$ genes, favoring the idea that AID acts downstream of the DNA lesions to mediate error prone processing.
\end{abstract}

Keywords: Activation induced cytidine deaminase (AID); Class switch recombination (CSR); DNA Double-Strand Break (DSB); Immunoglobulin lambda (Ig $\lambda$ ); Somatic hypermutation (SHM)

\section{INTRODUCTION}

Somatic hypermutation (SHM) of B cell Immunoglobulin (Ig) variable region genes and class switch recombination (CSR) in the Ig constant region genes allow further diversification of the primary antibody repertoire and increase the efficacy of the immune response. SHM and CSR are processes that: (a) take place in B cells of the GC (Neuberger and Milstein, 1995) (b) require the activation induced cytidine deaminase (AID) (Muramatsu et al., 2000) (c) are associated with DNA lesions (single strand and double-stranded breaks) (Bross et al., 2000; Papavasiliou and Schatz, 2000) (Kong and Maizels, 2001) and (d) are dependent on the transcription of the Ig-V region (Jacobs et al., 1999) and the -switch regions (Honjo et al., 2002), respectively. While point mutations and small deletions are found in the V (Goossens et al., 1998) as well as switch regions (Petersen et al., 2001; Nagaoka et al., 2002), the termination of a CSR involves deletion of a large fragment of intervening DNA between two active switch regions. In contrast to SHM, CSR involves the DNA dependent protein kinase (DNA PKcs) an enzyme involved in non-homologous end-joining
(Bemark et al., 2000) (for review, see also Honjo et al., 2002). Thus while previously the molecular mechanisms leading to CSR and SHM appeared to be distinct, the above mentioned parallels (see also Table I) favor a common mechanism in the initiation of CSR and SHM.

Mutations in the GC specific enzyme AID are causative for the autosomal recessive form of the human hyper Ig M syndrom 2 (HIGM2)(Revy et al., 2000). AID controls not only SHM and CSR (Muramatsu et al., 2000) but, as was shown recently in DT40 B cells, also gene conversion in avian Ig genes (Arakawa et al., 2002). As AID can mediate CSR of a recombination substrate in fibroblasts (Okazaki et al., 2002) and hybridomas (Martin et al., 2002), AID seems to be the only GC specific factor required for the induction of SHM and CSR. The close homology of AID to the RNA editing enzyme APOBEC-1 (Muramatsu et al., 1999) suggests that AID might be involved in RNA editing. Given this homology and the fact that APOBEC-1 requires the Apobec-1 Complementation Factor (ACF) (Lellek et al., 2000; Mehta et al., 2000 ) to deaminate $C^{6666}$ of the apolipoprotein $B$ mRNA to Uracil, AID might also function together with an ACFlike factor in RNA-editing. However, as shown in vitro,

*Corresponding author. Tel.: +31-0-43-38-8-2114. Fax: +31-0-43-38-8-4164. E-mail: h.jacobs@immuno.unimaas.nl 
TABLE I Comparing the features of SHM and CSR. See reviews: Jacobs and Bross (2001) and Honjo et al. (2002)

\begin{tabular}{lll}
\hline & \multicolumn{1}{c}{ SHM } & \multicolumn{1}{c}{ CSR } \\
\hline AID & Required & Required \\
DNA strand breaks & Occur & Occur \\
Transcription & Dependent & Dependent \\
Point mutations & Yes & Yes \\
Small deletions & Yes & Yes \\
$\begin{array}{l}\text { Deletion of large } \\
\text { DNA regions }\end{array}$ & No & Between two active \\
Non-homologous & Independent & Switch regions \\
end joining & & Generally required to terminate \\
& & the deletion process \\
\hline
\end{tabular}

AID deaminates deoxy-cytidine (Muramatsu et al., 1999), implicating two principle models of AID to function, a "RNA substrate model" and a "DNA substrate model", respectively. In the "DNA substrate model", AID acts within a nucleoprotein complex to deaminate deoxycytidines within ssDNA of transcribed $\mathrm{V}$ and switch regions. As shown in Fig. 1a, the presence of deoxy-uracil leads to a recruitment of the base excision repair machinery, causing nicks and/or staggered breaks which might become processed in an error prone fashion. This model suggests that DNA-DSBs normally associated with SHM of Ig V region genes are a direct product of AID action. Alternatively, AID might function as its closest homologue APOBEC-1 by editing a mRNA encoding a SHM/CSR control factor. This control factor might either induce the generation of DNA-DSBs or be involved at a post-cleavage repair step of the DNA-DSBs associated with SHM and CSR (Fig. 1b). Given the absolute requirement of AID in SHM, CSR and gene conversion, knowledge of AID function will provide the key information to understand how GC B cells are enabled, to increase the affinity of the antigen specific Ig pool, change the effector/homing function by isotype switching, and diversify the avian Ig repertoire by gene conversion. Identifying the system that changes the mutation rate from $10^{-9}$ (spontaneous mutation rate) to about $10^{-3}$ base pairs per generation in a higher eukaryotic cell is the focus of SHM research. This manuscript summarizes our findings on the role of transcription and the occurrence as well as the distribution of DNA-DSBs in hypermutating Ig genes of AID proficient and AID deficient GC B cells (Fukita et al., 1998; Bross et al., 2000). (a)

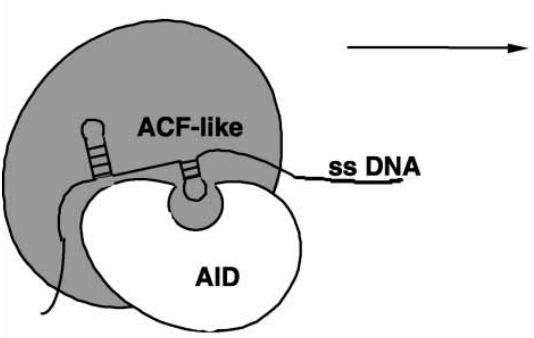

error prone fill in

error prone processing of DNA ends

(b)

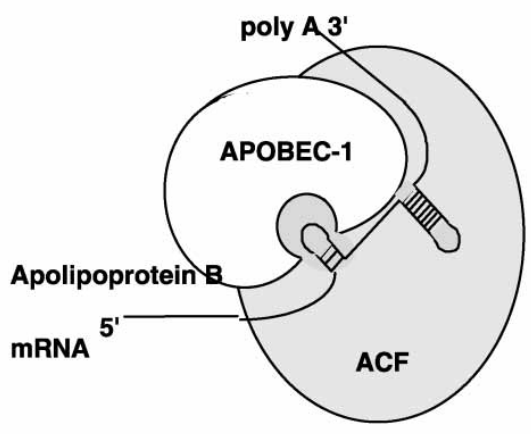

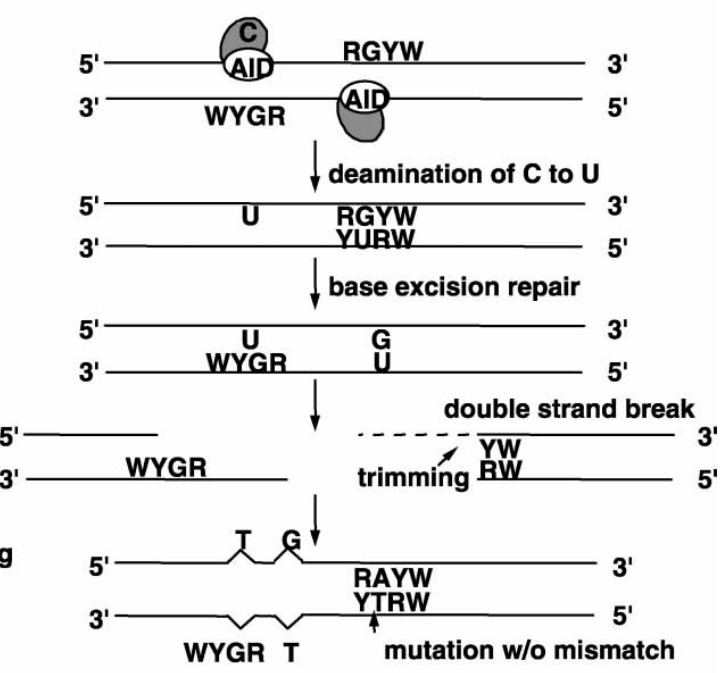

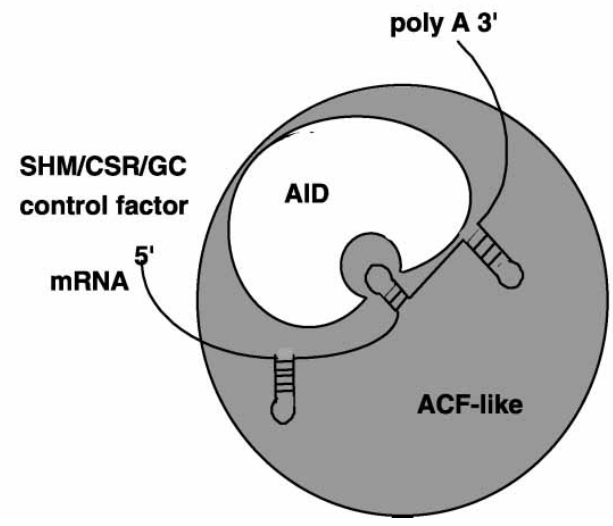

FIGURE 1 (a) "DNA-Substrate Model" (see text) (Jacobs and Bross, 2001). (b) "RNA-Substrate Model" (see text) (Jacobs and Bross, 2001). 


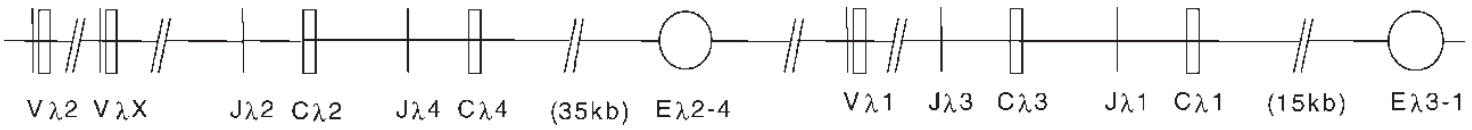

FIGURE 2 Genomic organization of the mouse $\operatorname{Ig} \lambda$ locus and $\lambda$ LC usage (see text).

\section{RESULTS AND DISCUSSION}

We and others have previously demonstrated the frequent occurrence of DNA-DSBs or nicks in hypermutating Ig genes (Jacobs and Bross, 2001). The introduction of DNA-DSBs is favored by transcription and consistent with the mutations in hypermutated $\mathrm{V}$ region genes, about $60 \%$ were found in RGYW/WRCY $(\mathrm{R}=\mathrm{A}$ or $\mathrm{G}, \mathrm{Y}=\mathrm{C}$ or $\mathrm{T}, \mathrm{W}=\mathrm{A}$ or $\mathrm{T})$. The $\mathrm{RGYW}$ motif and its inverse complement WRCY are hot spots intrinsic to the hypermutation mechanism. While these data clearly demonstrated the presence of breaks in rearranged $\mathrm{Ig} \mathrm{V}$ regions, the situation of non-rearranged i.e. germline (gl) V genes in GC B cells has not been addressed so far. This question is particularily interesting in view of the fact that normally rearranged $\mathrm{V}$ genes mutate at a higher frequency as germline (gl) $\mathrm{V}$ genes (Azuma, 1998). To address this issue, we have made use of Ig kappa ( $\kappa$ ) deficient mice, where only B cells expressing a functionally rearranged Ig lambda $(\lambda)$ light chain (LC) can develop (Zou et al., 1993). A schematic outline of the mouse $\operatorname{Ig} \lambda$ LC locus is shown in Fig. 2. The murine $\operatorname{Ig} \lambda$ LC locus likely arose by gene duplication and comprises two functional $\mathrm{V}$ genes $(\mathrm{V} \lambda 1$ and $\mathrm{V} \lambda 2$ ) and a pseudogene $(\mathrm{V} \lambda \mathrm{X}) . \mathrm{V} \lambda 1$ is preferentially rearranged to $\mathrm{J} \lambda 3$ and less frequently to $\mathrm{J} \lambda 1$ and more than $90 \%$ of $\operatorname{Ig} \lambda$ expressing B cells make use of $V \lambda 1$ rather than $V \lambda 2 . V \lambda 2$ is rearranged preferentially to $\mathrm{J} \lambda 2$ and less frequently to $\mathrm{J} \lambda 4$. The duplication of the $\lambda$ locus also included the enhancer giving rise to two independently active $\lambda$ enhancers (E $2-4$ and E $\lambda 3-1)$. While one enhancer activates one promotor at a given timepoint, two autonomous enhancers should activate two promotors e.g. the promotor of a rearranged as well as a non-rearranged $\mathrm{V} \lambda 1$ gene as present in most $\operatorname{Ig} \lambda$ positive $\mathrm{B}$ cells. This situation provides an ideal system to investigate the occurrence, frequency and distribution of DNA-DSBs in germline versus rearranged $\mathrm{V} \lambda$ genes of GC B cells. Therefore, AID proficient, $\kappa$ deficient mice and AID deficient mice were immunized. Ten days later GC B cells $\left(\mathrm{PNA}^{\text {high }}, \mathrm{CD} 19^{+}\right.$, Propidium Iodide ${ }^{-}\left(\mathrm{PI}^{-}\right)$,

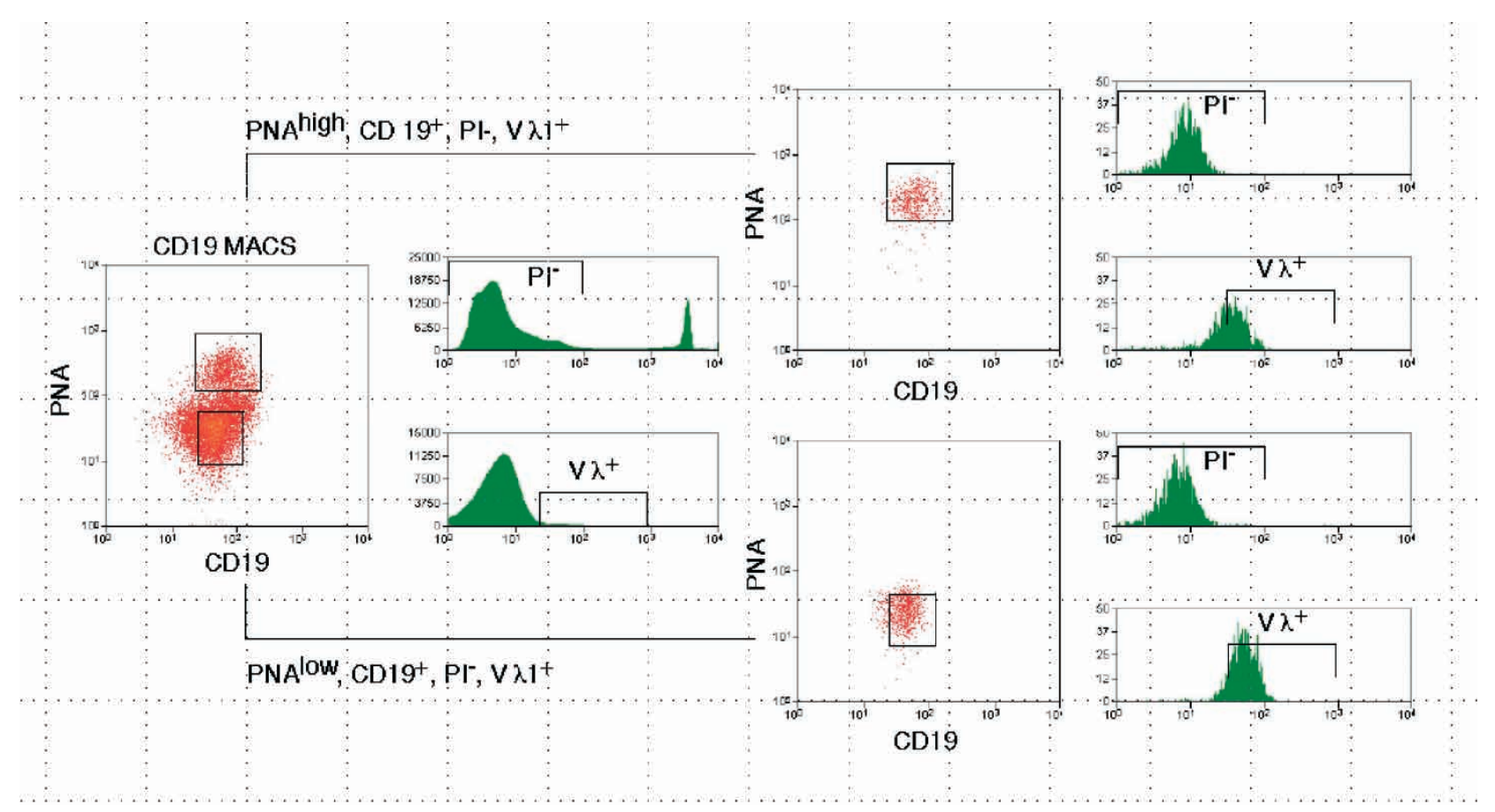

FIGURE 3 Sorting of $\mathrm{V} \lambda 1 / 2^{+} \mathrm{GC}$ and non-GC B cells: Splenic B cells were positively enriched by magnet activated cell sorting (MACS) using CD19specific beads. AID-deficient B cells are shown as an example. Hereafter cells were stained with a FITC-conjugated CD19 specific monoclonal antibody (clone 1D3), a PE-conjugated V $\lambda 1 / 2$ specific monclonal antibody (clone LS136), and biotinylated peanut agglutinin (PNA). Biotinylated PNA was revealed indirectly with Streptavidin-Allophycocyanin (SA-APC, Molecular Probes). GC-B cells (PNA ${ }^{\text {high }}$ ) in which SHM is known to be ongoing have compared to non-GC B cells (PNA ${ }^{\text {low }}$ ) more binding sites for peanut agglutinin (PNA). Dead cells were excluded by propidium iodine staining

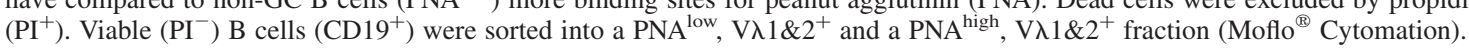




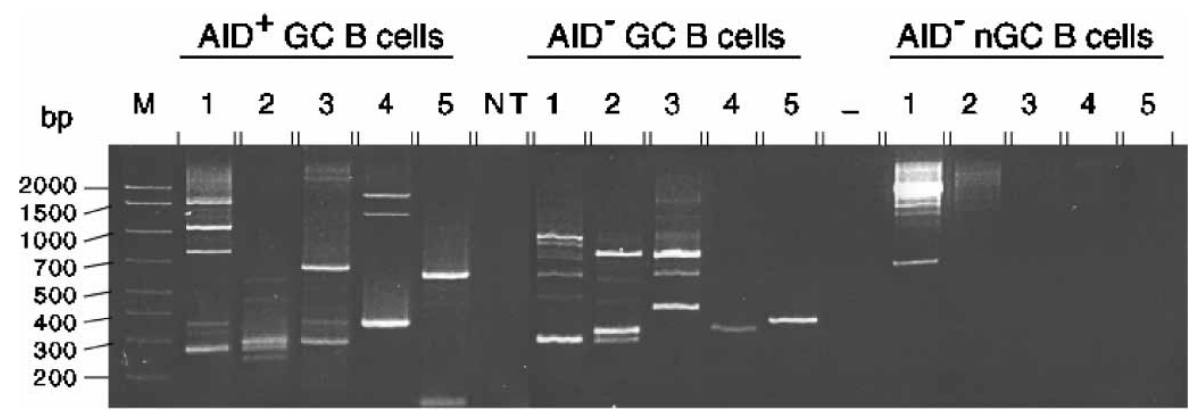

FIGURE 4 DNA-DSBs in the Ig $\lambda$ locus of GC B cells derived from AID proficient, Igк k.o. mice (AID ${ }^{+/+}$) and AID deficient mice (AID ${ }^{-/-}$). (a) Detection of DNA-DSBs. LM-PCR products of the $5^{\prime}$ break sites from 10000 (1), 5000 (2), 2500 (3), 1250 (4) and 625 (5) cell-equivalents of GC B cells $\left(\mathrm{CD} 19^{+}, \mathrm{PI}^{-}, \mathrm{PNA}^{\text {high }}\right)$ and non-GC B cells $\left(\mathrm{CD} 19^{+}, \mathrm{PI}^{-}, \mathrm{PNA}^{\text {low }}\right)$. LM-PCR products were separated on a $2 \%$ (w/v) TAE agarose gel and visualized with ethidium bromide under UV-light. The DNA size markers are indicated in base pairs. As revealed by Southern-blotting and sequencing, most PCR products are V $\lambda 1 \& 2$ specific.

$\mathrm{V} \lambda 1 / 2^{+}$) and non GC B cells $\left(\mathrm{PNA}^{\text {low }}, \mathrm{CD} 19^{+}, \mathrm{PI}^{-}\right.$and $\mathrm{V} \lambda 1 / 2^{+}$) were sorted from the spleens of these mice using a highspeed cell sorter (an example is shown in Fig. 3). From the sorted AID proficient and AID deficient B cell fractions, high molecular weight DNA was isolated and aliquots corresponding to a defined number of cells were ligated to blunt ended DNA adapters (splinkerettes) (Bross et al., 2000). The V $\lambda$ /adapter hybrids were PCR amplified in two rounds. The two primer sets anneal specifically within the $5^{\prime}$ region of $\mathrm{V} \lambda 1 \& 2$ genes and to the complement of the long strand of the splinkerette, respectively (Bross et al., 2000). If DNA-DSBs exist in the V $\lambda 1 \& 2$ region of hypermutating B cells, the PCR products should occur within the hypermutation domain. The $5^{\prime}$ boundary of the hypermutation domain lies downstream of the promoter transcription initiation site, increases rapidly along the leader and $\mathrm{V}(\mathrm{D}) \mathrm{J}$ exon and decreases beyond the $\mathrm{J}$ region. As previously found in targeted $\mathrm{V}_{\mathrm{H}} \mathrm{B} 1-8$ genes (Bross et al., 2000), distinct V $\lambda$ specific PCR products were derived from hypermutation competent GC B cells and only infrequently in small, non GC B cells (Fig. 4) (Bross et al., 2002). Southern-blot analysis with a radiolabeled $\mathrm{V} \lambda$ probe and sequencing revealed the specificity of nearly all PCR products (data not shown). The identity, location and site preference of DNA-DSBs in the $\operatorname{Ig} \lambda$ locus were determined by sequencing the cloned PCR products. DNA-DSBs are found in a region of 100-2000 base pairs downstream of rearranged $V \lambda 1 \& 2$ genes and interestingly at a similar frequency in non-rearranged, i.e. germline configured V $\lambda 1 \& 2$ segments of the $\lambda$ LC locus (Fig. 5a+b). Fifty-seven percent (43/76) of all DNADSBs lie within a RGYW/WRCY motif even though only $38 \%$ of randomly distributed DNA-DSBs are expected to occur at these sites, indicating a preference of DNA-DSBs to occur in RGYW/WRCY motif. A web supplement showing the exact location of the breaks has been published elsewhere (Bross et al., 2002).

The presence of a $\mathrm{J} \lambda$ element or a recombination signal sequence (RSS) at the $3^{\prime}$ end of $\mathrm{V} \lambda$ segments allows to distinguish between DNA-DSBs in rearranged versus germline $\mathrm{V} \lambda$ genes. Excluding the DNA-DSBs within V $\lambda$ segments, the relative frequency of DNA-DSBs in rearranged versus germline Ig $\lambda$ gene segments can be determined. Of the remaining 29 DNA-DSBs, $48 \%$ $(14 / 29)$ of the DNA-DSBs were found downstream of rearranged and 52\% (15/29) downstream of germline V $\lambda 1$ gene segments. As transcription favors the generation of DNA-DSBs and each of the two autonomous $\operatorname{Ig} \lambda$ enhancers, E $\lambda 2-4$ and $E \lambda 3-1$ can independently activate transcription of rearranged and non-rearranged $V \lambda$ genes, a high frequency of DNA-DSBs in germline $\mathrm{V} \lambda$ gene segments is expected. Regarding DNA-DSBs in V $\lambda 2$ gene segments, only $14 \%(2 / 14)$ are found downstream of rearranged and $86 \%(12 / 14)$ downstream of germline V $\lambda 2$ gene segments. This finding likely relates to the fact that VJ rearrangements at the $\operatorname{Ig} \lambda$ locus of B cell precursors preferentially $(>90 \%)$ make use of $\mathrm{V} \lambda 1$ segments, leaving most V $\lambda 2$ alleles $(>90 \%)$ in germline configuration. In this context it should be mentioned, that according to the enhancer flip flop model (Wijgerde et al., 1995) a single enhancer suffices to activate sequentially several promoters. Therefore, DNA-DSBs in germline $\mathrm{V}_{\mathrm{H}}$ or Vk gene segments are also expected to be introduced, albeit taking the cooperation between the intronic and $3^{\prime}$ enhancers at the IgHC and Igk LC locus and the distance to upstream $\mathrm{V}$ gene promoters into account at lower frequency.

Comparing AID proficient and AID deficient GC B cells no obvious qualitative nor quantitative differences with regard to DNA-DSBs were found. Thus, regarding the majority of DNA-DSBs, AID deficiency appears not to affect the generation, frequency (number of PCR products) and distribution of DNA-DSBs (size range of PCR products) along the rearranged and germline $V \lambda 1 \& 2$ genes of GC B cells in our assay (Figs. 4, 5b). Again, considering the 19 tetramers with a RGYW consensus in V $\lambda 1 \& 2$ segments, $38 \%$ of randomly distributed DNADSBs are expected to occur at these sites, 56\% (15/27) of all DNA-DSBs found in AID-deficient GC B cells locate at a RGYW consensus motif. As summarized in Figs. 4 
(a)

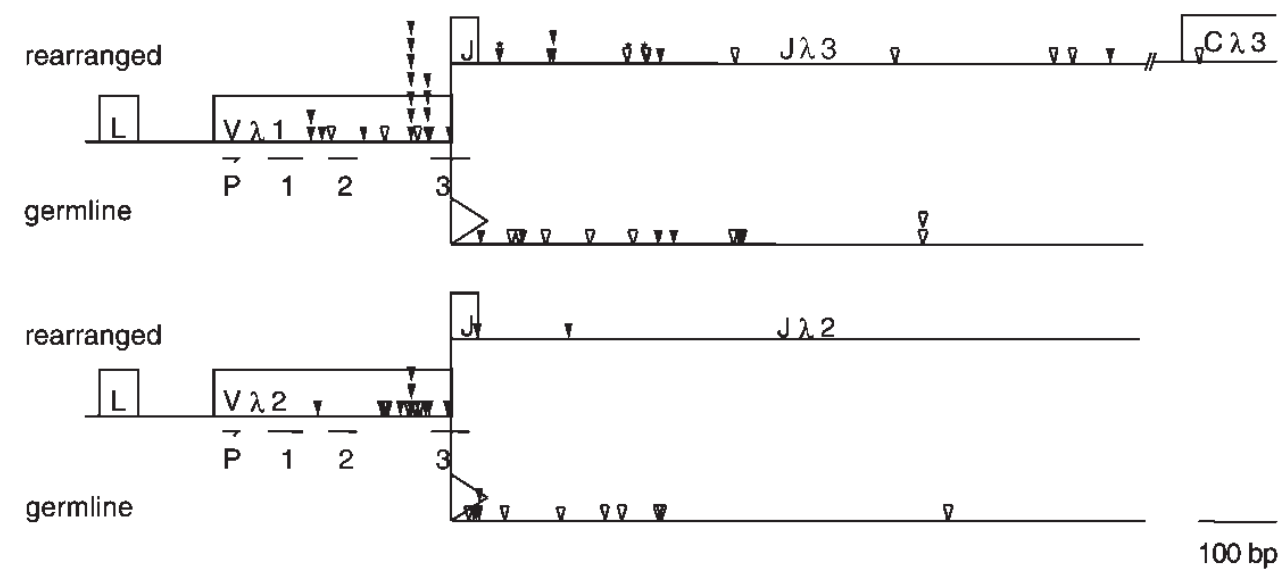

(b)
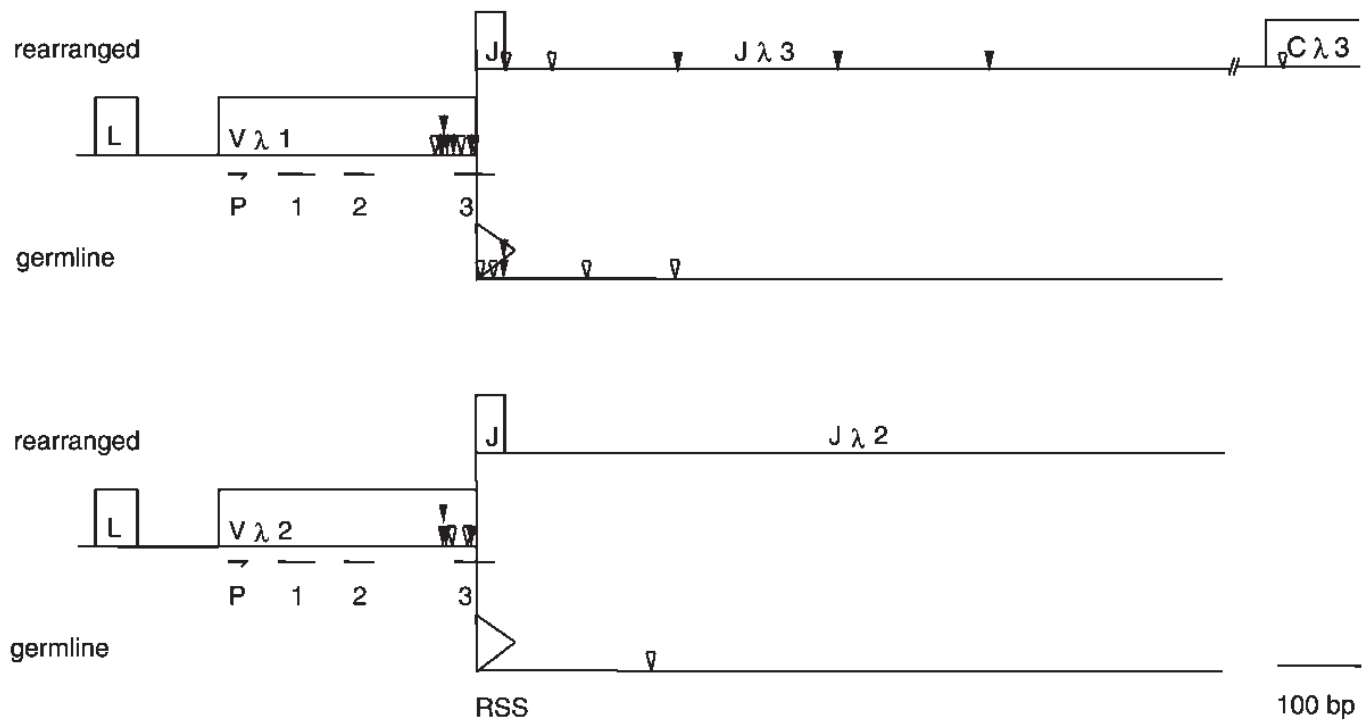

FIGURE 5 (a) DSBs in rearranged and germline V $\lambda$ gene segments of hypermutating Igk deficient GC B cells. The distribution and location of the DSBs found are depicted as arrows. Vertically aligned arrows indicate DSBs obtained from independent LM-PCR reactions. Closed arrows indicate DNA-DSBs at an RGYW motif, open arrows indicate DNA-DSBs outside an RGYW motif. P $=1$ location of the V $\lambda$ internal primer. $1,2,3=1$ location of the complementary determining regions (CDR1, 2 and 3 respectively). Asterisks above arrows indicate DSBs in V $\lambda 1 \mathrm{~J} \lambda 3$ rearranged $\lambda$ light chain genes. Triangles indicate the RSS. (b) DSBs in the Ig $\lambda$ locus of AID deficient GC B cells and non-GC B cells. (i) AID does not control the generation of DSBs in hypermutating Ig genes derived from the amplification of the $5^{\prime}$ break sites in V $\lambda 1 \& 2$ gene segments from 10000 (1), 5000 (2), 2500 (3), 1250 (4) and 625 (5) cell-equivalents of GC B cells $\left(\mathrm{V} \lambda+, \mathrm{CD}^{+} 9^{+}, \mathrm{PI}^{-}, \mathrm{PNA}^{\text {high }}\right)$ from AID ${ }^{+/+}$and AID ${ }^{-1-}$ mice as well as non-GC B cells $(\mathrm{V} \lambda+$, $\mathrm{CD}_{1}{ }^{+}, \mathrm{PI}^{-}, \mathrm{PNA}^{\text {low }}$ ) from AID deficient mice (ii) DSBs in rearranged and non-rearranged V $\lambda$ genes of GC B cells from AID deficient mice (see text). For annotations see 5(a).

and $5 \mathrm{~b}$, the frequency, distribution as well as the preference of DNA-DSBs to occur in RGYW motifs appear not to be controlled by AID (The exact location of the breaks can be found as a web supplement within Bross et al. (2002)).

If rearranged and germline-configured, $\mathrm{V} \lambda$ genes are equal substrates of an unknown nuclease, and are also equally targeted by the SHM system? To (re)-address this question in our system the mutation frequencies of rearranged and non-rearranged $\mathrm{V} \lambda 1$ genes were determined by amplifying and sequencing these regions from single class-switched $\mathrm{V} \lambda 1^{+}, \mathrm{CD} 19^{+}, \operatorname{Ig} \mu \delta$ memory $\mathrm{B}$ cells isolated from the spleen of $\mathrm{C} 57 \mathrm{~B} 1 / 6$ mice
(Bross et al., 2002). Despite the fact that DNA-DSBs are found at an equal frequency in rearranged and germline $\mathrm{V} \lambda 1$ genes, the frequency of mutations differs. While $57 \%(42 / 74)$ of rearranged $\mathrm{V} \lambda 1$ genes were mutated at a frequency of $0.61 \%$ (144 mutations in 23680 base pairs sequenced), only $21 \%$ (7/34) of germline $\mathrm{V} \lambda 1$ genes sequenced were mutated at a frequency of $0.11 \%$ (12 mutations in 10880 base pairs sequenced). Taking into account that SHM has been active in 57\% of the cells, the actual mutation frequency is $1.07 \%$ for rearranged and $0.19 \%$ for germline $\mathrm{V} \lambda 1$ genes. In line with previous studies, about $60 \%$ of class switched $\mathrm{V} \lambda 1^{+}, \mathrm{CD} 19^{+}, \operatorname{Ig} \mu \delta$ memory $\mathrm{B}$ cells have a mutated, rearranged $\mathrm{V} \lambda 1$ gene 
(Fukita et al., 1998; Jacobs et al., 1998) and mutations in germline configured $\mathrm{V} \lambda$ segments occur, albeit at a significantly lower frequency (Azuma, 1998). Therefore, although DNA-DSBs are introduced at similar frequencies in germline and rearranged $\mathrm{V} \lambda 1 \& 2$ genes, SHM is preferentially targeted to the rearranged $\mathrm{V} \lambda 1 \& 2$ genes, suggesting that a DNA-DSB itself does not suffice for optimal targeting of the hypermutation machinery. We propose that usually most DNA-DSBs are efficiently repaired in a non-mutagenic manner and therefore, do not lead to cell death. In the presence of AID, however, the likelyhood that the processing of the lesions (nicks or DNA-DSBs) becomes error prone increases, and the risk of disfavored mutations potentially leading to cell death or abberant development, like lymphomas and autoimmunity.

In conclusion, despite the fact that DNA-DSBs are found at similar frequencies in germline and rearranged $\mathrm{V} \lambda$ gene segments, SHM is preferentially targeted to rearranged $\mathrm{V} \lambda$ gene segments. As the generation of DNA-DSBs is dependent on transcription, or at least on the initiation of transcription, the occurrence of DNADSBs in germline and rearranged $\mathrm{V} \lambda$ genes at a similar frequency likely relates to the presence of two independent $\lambda$ enhancers. The data clearly show that most of the DNA-DSBs are AID independent. Therefore three possibilities have been raised (Bross et al., 2002); (1) none of the DNA-DSBs are involved in SHM, (2) only a small fraction of DNA-DSBs are involved in SHM and (3) DNA-DSBs are generated in transcription dependent but AID independent manner. The latter possibility suggests that AID function was established to utilize these lesions in order to mediate Ig gene remodeling like CSR, SHM or gene conversion. Present studies aim on the identification of the nuclease and the identification of the AID substrate.

\section{MATERIAL AND METHODS}

\section{Mice}

The generation and genotyping of AID- and Igк-deficient mice have been described elsewhere (Zou et al., 1993; Muramatsu et al., 2000).

\section{Immunization}

Igк K.O. mice were immunized with $0.2 \mathrm{ml}$ of a $10 \%$ sheep red blood cells solution in PBS (Bross et al., 2000). AID K.O. mice were immunized with NP-CG. For the immunization with NP-CG, NP(28)-CGG ${ }^{\circledR}$ (Biosearch Technologies Inc.) is resuspended at $1 \mathrm{mg} / \mathrm{ml}$ in PBS, an equal volume of Alu-Gel-S ${ }^{\circledR}$ (Serva) is added, mixed, incubated over night at $4^{\circ} \mathrm{C}$, and $0.2 \mathrm{ml}$ of this suspension (100 $\mu \mathrm{g} \mathrm{NP(28)-CGG)} \mathrm{is} \mathrm{injected} \mathrm{i.p.} \mathrm{For} \mathrm{the} \mathrm{analysis}$ of DNA-DSBs mice were sacrificed 7 days after immunization, for the analysis of SHM, mice were sacrificed 10 days after immunization.

\section{Cell Sorting, and DNA Isolation}

Sorting of GC and non-GC B cells was done using a combination of magnet activated cell sorting (MACS) (Miltenyi Biotec) and fluorescence activated cell sorting (FACS) using a FACStar ${ }^{\mathrm{TM}}$ (Becton Dickinson). The isolation of high molecular weight DNA from these fractions has been described elsewhere (Ref). Sorting of V $\lambda 1 \& 2$ expressing GC and non-GC B cell subsets was achieved with a $\mathrm{V} \lambda 1 \& 2$ specific monoclonal antibody (clone LS136) as described in Jacobs et al. (1998).

\section{Analysis of SHM in Germline Vג1 Gene Segments}

For the amplification of germline $\mathrm{V} \lambda 1$ gene segments a semi-nested PCR assay was applied using the reverse $\mathrm{V} \lambda 1$ intron primer in combination with the $V \lambda 1 \& 2$ external primer for the 1 st round and the $\mathrm{V} \lambda 1 \& 2$ internal primer for the 2nd round of PCR amplification. The PCR amplification was performed as described (Jacobs et al., 1998).

\section{Amplification, Cloning and Sequencing of Splinkerette-ligated V $\boldsymbol{\lambda}$ Genes}

The ligation of the splinkerettes has been described elsewhere (Jacobs et al., 1998). The quantity of the DNA used is based on a defined number of sorted cells. Based on previous experiments and as determined by semiquantitative $\mathrm{PCR}$ reactions with $\mathrm{Ku} 70$ specific primers (see below) this method of DNA quantification is reproducible. Specific amplification of adapter-ligated V $\lambda 1 \& 2$ genes from genomic DNA was achieved by using a nested PCR strategy. In the first round, the external splinkerette primer was used in combination with the external $\mathrm{V} \lambda$ primer. For the second round of amplification the internal splinkerette primer was used in combination with the internal $\mathrm{V} \lambda$ primer. To detect any $\mathrm{V} \lambda$ /adapter hybrids, we used the same PCR conditions as described for the amplification of the $\mathrm{V} \lambda 1$ genes from single cells (Jacobs et al., 1998). PCR products were resolved on a $2 \%$ (w/v) agarose gel, visualized with ethidium bromide under UV-light and isolated from agarose gel slices using the QiaQuick ${ }^{\circledR}$ matrix (Qiagen). After isolation the PCR products were cloned into the TOPO $\mathrm{pCRII}^{\circledR}$ vector from the TOPO TA Cloning ${ }^{\circledR}$ kit (Invitrogen) and sequenced using the DyeDeoxy Terminator Cycle Sequencing kit ${ }^{\circledR}$ (Applied Biosystems).

\section{Oligonucleotides}

V $\lambda 1 \& 2$ external primer: 5'-GGGTATGCAACAATGCGCATCTTGTC-3', V $\lambda 1 \& 2$ internal primer: 5'-GCGAAGCGAAGAGAAGCTTGTGACTCAGGAATCTGCA-3', 
$\mathrm{V} \lambda 1$ intron primer 5'-AATGATTCTATGTTCTGCCAAGTC-3'. External splinkerette primer: 5'-CGAAGAGTAACCGTTGCTAGGAGAGACC- $3^{\prime}$. Internal splinkerette primer: 5'-GTGGCTGAATGAGACTGGTGTCGAC-3'. Splinkerette oligomers: 5'CGAAGAGTAACCGTTGCTAGGAGAGACCGTGGCTGAATGAGACTGGTGTCGACACTAGTGG-3' (long strand, 61-mer), 5'-CCACTAGTGTCGACACCAGTCTCTAATTTTTTTTTTCAAAAAAA-3' (short strand, 44-mer), 5'ACACGGCTTCCTTAATGTGA-3' (KU70 forward primer), and 5' GGCTGGCTTTAGCACTGTCA (KU70 reverse primer).

\section{Acknowledgements}

The authors like to thank Sue Cooper and Roy Allenspach for expert technical assistance, Tracy Hayden and Hubertus Kohler for fluorescence activated cell sortings, Erwin Schilliger for preparing figures, the BII animal caretaker team for their biotechnical help, and F. McBlane and G. Kline for critically reading the manuscript. Many thanks to Drs Sigfried Weiss and Holger Engel at the GBF in Braunschweig, Germany for providing valuable sequence information on the mouse $\operatorname{Ig} \lambda$ locus and to F. Hoffmann La Roche Ltd, Basel, Switzerland, which founded and supported the Basel Institute for Immunology.

\section{References}

Arakawa, H., Hauschild, J. and Buerstedde, J.M. (2002) "Requirement of the activation-induced deaminase (AID) gene for immunoglobulin gene conversion", Science 295, 1301-1306.

Azuma, T. (1998) "Somatic hypermutation in mouse lambda chains", Immunol. Rev. 162, 97-105.

Bemark, M., Sale, J.E., Kim, H.J., Berek, C., Cosgrove, R.A. and Neuberger, M.S. (2000) "Somatic hypermutation in the absence of DNA-dependent protein kinase catalytic subunit (DNA-PKcs) or recombination-activating gene (RAG)1 activity", J. Exp. Med. 192, $1509-1514$.

Bross, L., Fukita, Y., McBlane, F., Demolliere, C., Rajewsky, K. and Jacobs, H. (2000) "DNA double-strand breaks in immunoglobulin genes undergoing somatic hypermutation", Immunity 13, 589-597.

Bross, L., Muramatsu, M., Kinoshita, K., Honjo, T. and Jacobs, H. (2002) "DNA double-strand breaks: prior to but not sufficient in targeting hypermutation", J. Exp. Med. 195, 1187-1192.

Fukita, Y., Jacobs, H. and Rajewsky, K. (1998) "Somatic hypermutation in the heavy chain locus correlates with transcription", Immunity $\mathbf{9}$, $105-114$.

Goossens, T., Klein, U. and Kuppers, R. (1998) "Frequent occurrence of deletions and duplications during somatic hypermutation: implications for oncogene translocations and heavy chain disease", Proc. Natl Acad. Sci. USA 95, 2463-2468.

Honjo, T., Kinoshita, K. and Muramatsu, M. (2002) "Molecular mechanism of class switch recombination: linkage with somatic hypermutation", Аnпи. Rev. Immunol. 20, 165-196.
Jacobs, H. and Bross, L. (2001) "Towards an understanding of somatic hypermutation", Curr. Opin. Immunol. 13, 208-218.

Jacobs, H., Fukita, Y., van der Horst, G.T., de Boer, J., Weeda, G., Essers, J., de Wind, N., Engelward, B.P., Samson, L., Verbeek, S., de Murcia, J.M., de Murcia, G., te Riele, H. and Rajewsky, K. (1998) "Hypermutation of immunoglobulin genes in memory B cells of DNA repair- deficient mice", J. Exp. Med. 187, 1735-1743.

Jacobs, H., Puglisi, A., Rajewsky, K. and Fukita, Y. (1999) "Tuning somatic hypermutation by transcription", Curr. Top. Microbiol. Immunol. 246, 149-158.

Kong, Q. and Maizels, N. (2001) "DNA breaks in hypermutating immunoglobulin genes: evidence for a break- and-repair pathway of somatic hypermutation", Genetics 158, 369-378.

Lellek, H., Kirsten, R., Diehl, I., Apostel, F., Buck, F. and Greeve, J. (2000) "Purification and molecular cloning of a novel essential component of the apolipoprotein B mRNA editing enzyme-complex", J. Biol. Chem. 275, 19848-19856.

Martin, A., Bardwell, P.D., Woo, C.J., Fan, M., Shulman, M.J. and Scharff, M.D. (2002) "Activation-induced cytidine deaminase turns on somatic hypermutation in hybridomas", Nature 415, 802-806.

Mehta, A., Kinter, M.T., Sherman, N.E. and Driscoll, D.M. (2000) "Molecular cloning of apobec-1 complementation factor, a novel RNA- binding protein involved in the editing of apolipoprotein B mRNA", Mol. Cell. Biol. 20, 1846-1854.

Muramatsu, M., Sankaranand, V.S., Anant, S., Sugai, M., Kinoshita, K., Davidson, N.O. and Honjo, T. (1999) "Specific expression of activation-induced cytidine deaminase (AID), a novel member of the RNA-editing deaminase family in germinal center B cells", J. Biol. Chem. 274, 18470-18476.

Muramatsu, M., Kinoshita, K., Fagarasan, S., Yamada, S., Shinkai, Y. and Honjo, T. (2000) "Class switch recombination and hypermutation require activation-induced cytidine deaminase (AID), a potential RNA editing enzyme", Cell 102, 553-563.

Nagaoka, H., Muramatsu, M., Yamamura, N., Kinoshita, K. and Honjo, T. (2002) "Activation-induced deaminase (AID)-directed hypermutation in the immunoglobulin Smu region: implication of AID involvement in a common step of class switch recombination and somatic hypermutation", J. Exp. Med. 195, 529-534.

Neuberger, M.S. and Milstein, C. (1995) "Somatic hypermutation", Curr. Opin. Immunol. 7, 248-254.

Okazaki, I.M., Kinoshita, K., Muramatsu, M., Yoshikawa, K. and Honjo, T. (2002) "The AID enzyme induces class switch recombination in fibroblasts", Nature 416, 340-345.

Papavasiliou, F.N. and Schatz, D.G. (2000) "Cell-cycle-regulated DNA double-stranded breaks in somatic hypermutation of immunoglobulin genes", Nature 408, 216-221.

Petersen, S., Casellas, R., Reina-San-Martin, B., Chen, H.T., Difilippantonio, M.J., Wilson, P.C., Hanitsch, L., Celeste, A., Muramatsu, M., Pilch, D.R., Redon, C., Ried, T., Bonner, W.M., Honjo, T., Nussenzweig, M.C. and Nussenzweig, A. (2001) "AID is required to initiate Nbs1/gamma-H2AX focus formation and mutations at sites of class switching", Nature 414, 660-665.

Revy, P., Muto, T., Levy, Y., Geissmann, F., Plebani, A., Sanal, O., Catalan, N., Forveille, M., Dufourcq-Labelouse, R., Gennery, A., Tezcan, I., Ersoy, F., Kayserili, H., Ugazio, A.G., Brousse, N., Muramatsu, M., Notarangelo, L.D., Kinoshita, K., Honjo, T., Fischer, A. and Durandy, A. (2000) "Activation-induced cytidine deaminase (AID) deficiency causes the autosomal recessive form of the Hyper-IgM syndrome (HIGM2)", Cell 102, 565-575.

Wijgerde, M., Grosveld, F. and Fraser, P. (1995) "Transcription complex stability and chromatin dynamics in vivo", Nature 377, 209-213.

Zou, Y.R., Gu, H. and Rajewsky, K. (1993) "Generation of a mouse strain that produces immunoglobulin kappa chains with human constant regions", Science 262, 1271-1274. 


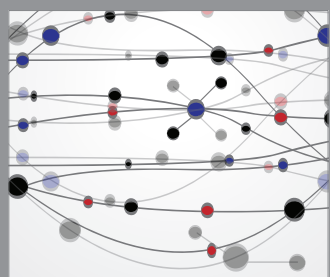

The Scientific World Journal
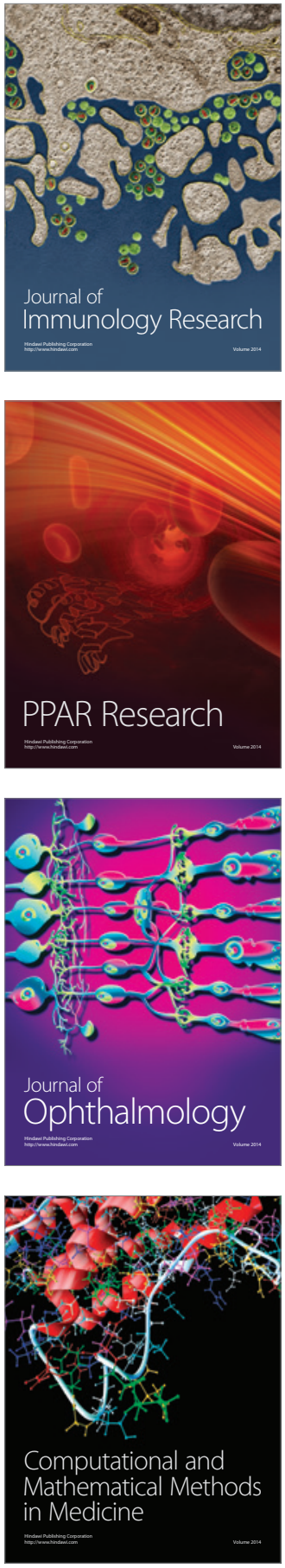

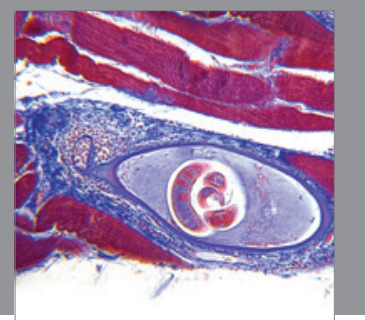

Gastroenterology

Research and Practice
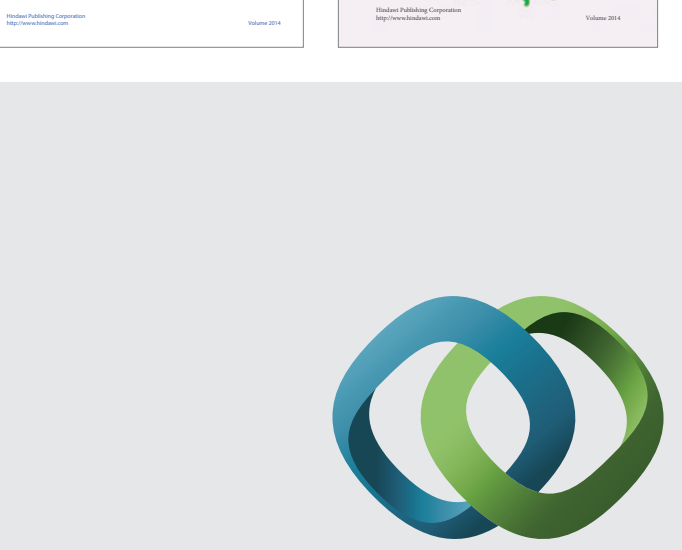

\section{Hindawi}

Submit your manuscripts at

http://www.hindawi.com
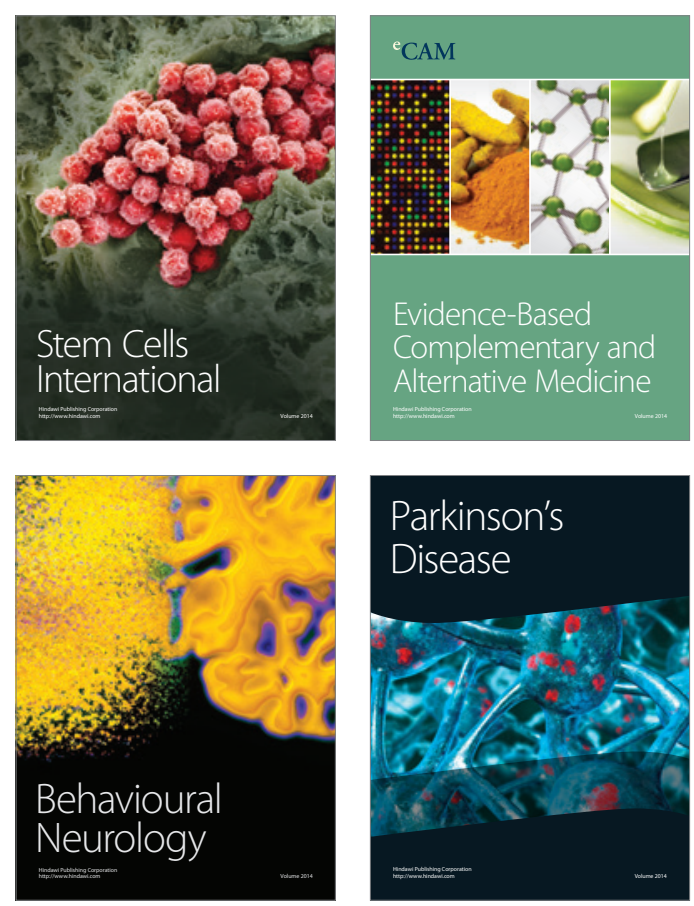

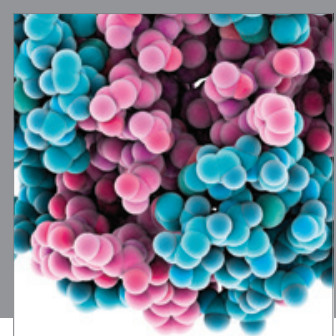

Journal of
Diabetes Research

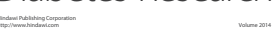

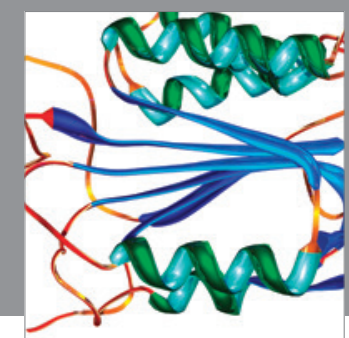

Disease Markers
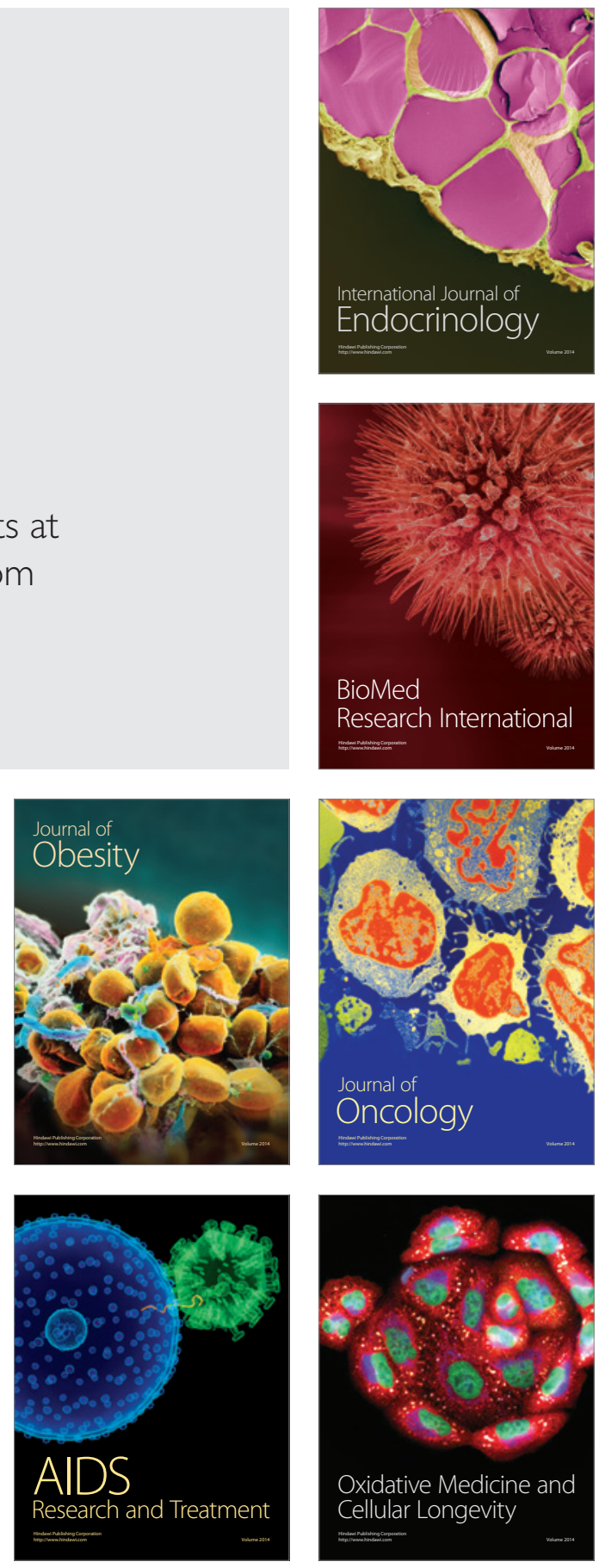